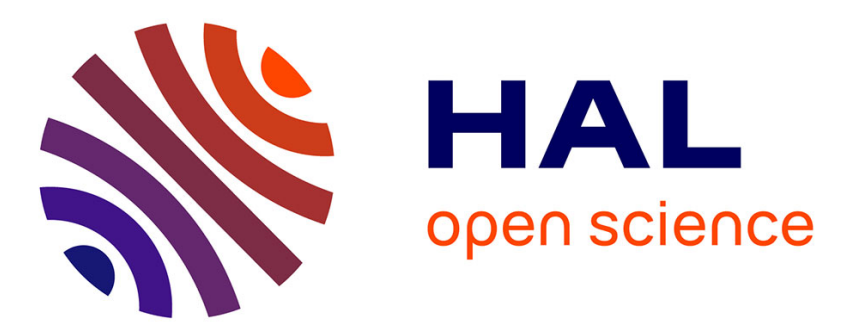

\title{
" État et perspectives de la lexicographie historique du français"
}

\author{
Jean-Paul Chauveau, Eva Buchi
}

\section{To cite this version:}

Jean-Paul Chauveau, Eva Buchi. "État et perspectives de la lexicographie historique du français ". Lexicographica. International Annual for Lexicography, 2011, 27, pp.101-122. 10.1515/lexi.2011.008 . halshs-00684490

\section{HAL Id: halshs-00684490 \\ https://shs.hal.science/halshs-00684490}

Submitted on 2 Apr 2012

HAL is a multi-disciplinary open access archive for the deposit and dissemination of scientific research documents, whether they are published or not. The documents may come from teaching and research institutions in France or abroad, or from public or private research centers.
L'archive ouverte pluridisciplinaire HAL, est destinée au dépôt et à la diffusion de documents scientifiques de niveau recherche, publiés ou non, émanant des établissements d'enseignement et de recherche français ou étrangers, des laboratoires publics ou privés. 


\title{
État et perspectives de la lexicographie historique du français Jean-Paul Chauveau \& Éva Buchi (ATILF, CNRS \& Nancy-Université)
}

\begin{abstract}
This paper ('State and perspectives of French historical lexicography') deals with the many kinds of excellent French historical dictionaries, be it general lexicography (FEW, DEAF, TLF-Étym), dictionaries dedicated to etymological classes (Arveiller's Orientalia, Baldinger's Etymologien), dictionaries for diatopically defined varieties (primary French dialects and regional varieties of French) or dictionaries for specialized vocabulary.
\end{abstract}

\section{Introduction}

La lexicographie historique française (historique étant ici entendu au sens fort de 'diachronique') dispose d'un grand nombre d'excellentes publications, qui touchent non seulement la lexicographie générale, mais aussi la lexicographie spécialisée. Dans ce qui suit, nous limiterons notre étude aux productions qui nous apparaissent comme les fleurons dans les différents domaines représentés, que cela concerne la lexicographie générale ou les dictionnaires centrés sur des ensembles étymologiques, des variétés diatopiques ou encore un secteur du lexique spécialisé (cf. aussi Buchi / Renders à paraître).

\section{Lexicographie générale}

\subsection{FEW}

Après la fin de la refonte de la tranche alphabétique A- (volumes 24 et 25) et de la publication des matériaux d'origine inconnue (volumes 21 à 23), les publications se sont d'abord donné pour but de faciliter la consultation et la lecture du FEW. Un index raisonné des formes (ATILF 2003) permet d'accéder beaucoup plus facilement à l'essentiel des matériaux contenus dans le FEW. Cet index a un caractère provisoire, dans l'attente d'une informatisation complète du FEW entier qui permettrait d'accéder à la totalité du matériel. La publication du Complément (Chauveau / Greub / Seidl 2010) a fourni une bibliographie mise à jour, qui donne la clé des nombreux sigles bibliographiques créés depuis la précédente édition qui datait de 1957. La métalangue est désormais le français et la liaison avec la lexicologie historique galloromane est assurée par la mention du correspondant de chaque sigle dans les bibliographies de référence du DEAF, pour l'ancien français, et du DOM, pour l'ancien occitan. La liste des comptes rendus de chacun des fascicules du FEW, de même que la liste des travaux critiques sur le FEW recensent la réception critique de l'ouvrage. Le projet d'informatisation actuellement à l'étude, après rétroconversion des articles (cf. Renders 2011), mettra en ligne l'ensemble de l'ouvrage et permettra différentes possibilités d'interrogation sur son contenu qui seront couplées avec les références bibliographiques et géolinguistiques complètes et un dispositif permettant de cartographier les données localisées.

La rédaction du dictionnaire proprement dit se poursuit par une refonte sélective de la tranche alphabétique B-. Il était devenu évident qu'il était impossible de continuer la refonte du premier volume du FEW, paru entre 1922 et 1928, sur le modèle de ce qui avait été fait pour la lettre A. Par rapport à la première rédaction, le volume des matériaux traités avait été largement décuplé, de même que la durée de rédaction. Refondre, dans des délais acceptables, l'entier de la lettre B sur cette même base aurait exigé des moyens humains impossibles à réunir. Entre les diverses autres possibilités, le choix s'est porté sur ce qui fait l'originalité de la pratique étymologique mise au point et portée à son plus haut degré d'accomplissement par von Wartburg, la synthèse galloromane. Les autres entreprises étymologiques en chantier dans le même domaine géolinguistique se concentrent sur un secteur géographique, ou sur un sociolecte, ou sur une catégorie de données, ou sur une période limitée de l'histoire linguistique. Seul le FEW se donne pour objectif de traiter l'histoire lexicale dans toutes ses ramifications et ses composantes. Son intérêt spécifique réside dans son refus des 
délimitations. Parce qu'il conjoint des données qui sont habituellement séparées, il donne des aperçus, et donc des liaisons et des possibilités d'explication, qui resteraient ignorés sans lui. Du fait qu'il s'interdit les angles morts, les zones d'ombre où s'alimentent les spéculations invérifiables reçoivent des clartés qui dissipent les erreurs et mettent en évidence des solutions non entrevues jusqu'ici. Il a donc été choisi d'appliquer la méthode wartburgienne là où elle atteint le maximum de ses potentialités, c'est-à-dire aux articles où sa méthodologie peut révéler toute sa force heuristique, des articles concernant tout le galloroman depuis ses débuts jusqu'à l'époque contemporaine et dans toutes ses variétés. Une cinquantaine de tels articles ont été retenus, qui n'excluent pas le traitement des autres membre de la même famille lexicale, au contraire: ainsi l'article BATĀRE s'écrit parallèlement à celui du dérivé BATĀCULĀRE. Le choix de la publication en ligne, sur le site de l'ATILF (Chauveau 2006-), a fait disparaitre les contraintes de pagination; le volume de tels articles peut dépasser les trois cent mille caractères et constituer une véritable monographie lexicale.

Du fait de l'orientation que lui a donnée von Wartburg, le FEW se situe d'emblée dans une perspective romane. Non seulement les données françaises sont traitées au sein d'un tableau galloroman, mais ce tableau lui-même est conçu comme un élément constitutif d'un panneau roman. Lorsque les explications reçues pour les données françaises et galloromanes s'avèrent ad hoc et impossibles à tenir, l'ensemble roman peut se révéler apte à fournir de nouvelles solutions. Ainsi les substantifs français féminins baisse 'dépression de terrain' et baissière 'dépression de terrain; ce qui reste au fond d'un tonneau' sont traditionnellement considérés comme un déverbal et un dérivé de baisser. Cependant on ne connaît pas d'autre substantif dérivé en -ier et -ière sur une base verbale: force est d'analyser baissière comme un dérivé de baisse. Et si les deux antonymes hausse 'action de monter' et baisse 'action de baisser' sont d'authentiques déverbaux, baisse 'dépression de terrain', qui leur est antérieur de près de cinq siècles, n'est pas la dénomination d'un affaissement de terrain, mais d'un lieu qui est naturellement bas, ce qui ne plaide pas pour un déverbal. La formation de tels lexèmes est tout à fait problématique dans cette perspective purement française. Pour trouver un modèle susceptible d'en rendre compte, il importe de prendre appui sur la grande ancienneté de ces types lexicaux et de chercher s'ils ne pourraient pas correspondre à une dérivation romane. De fait la concordance de plusieurs langues romanes a fait reconstruire une petite liste de substantifs féminins déadjectivaux romans en -ia, tels que, par exemple, ${ }^{*}$ crassia, à partir de crassus, *grossia à partir de grossus, *plania à partir de planus, etc. au sein de laquelle un *bassia n.f. 'ce qui est bas' dérivé de bassus adj. 'bas' s'intègre parfaitement et fournit le prototype régulier aussi bien du français baisse n.f. 'dépression de terrain' que de ses équivalents francoprovençal bési, occitan baisso et béarnais bache qu'il est déconseillé d'expliquer par quatre conversions parallèles. Dans le même ordre d'idées, l'étymon du français beugler v.intr. '(d'un bovidé) pousser le cri intense et prolongé qui lui est particulier, mugir' a pu être considéré comme un dérivé d'afr. mfr. bugle 'jeune bœuf', représentant semisavant du latin BūCULUS, ou bien comme le représentant semi-savant d'un latin tardif *BūCULĀRE 'mugir' dont le synonyme normand beuiller serait le résultat proprement héréditaire, ainsi que le fait le LEI $(7,1402)$, qui place ces formes au niveau du ladin bugolér 'mugir' et du frioulan bogolâ/bovolâ 'mugir'. La solution unitaire est plus économique que de multiples explications indépendantes. Si l'on veut trancher l'alternative, il importe de soumettre l'intégralité des données françaises aux deux hypothèses. Ces données présentent l'immense avantage de comporter plusieurs centaines d'attestations précisément localisables et chronologisables et appartenant à des systèmes linguistiques bien documentés et étudiés, ce qui fournit un terrain d'investigation particulièrement propice à la mise à l'épreuve des hypothèses. L'analyse et le classement de l'ensemble des types verbaux apparentés par la forme et le sens révèle qu'il s'agit soit des issues d'un type français b(e)ugler qui n'est pas attesté avant le $16^{\mathrm{e}}$ siècle, et qui donc peut difficilement être autre chose qu'un dérivé de 
bugle 'jeune bœuf', soit des résultats de ce verbe après croisement avec des verbes synonymes ou parasynonymes comme les représentants de MŪGILĀRE et de ULULĀRE. En fin de compte, les données françaises se laissent sans difficulté analyser comme des dérivés directs ou indirects d'afr. mfr. bugle 'jeune bœuf' et ne proposent aucun argument en faveur d'un latin tardif *BūCULĀRE 'mugir' dont les chances d'avoir existé s'amenuisent d'autant. Le choix de la perspective romane place les données gallo-romanes dans un éclairage qui leur est bénéfique ou dont bénéficient les autres langues romanes, selon les cas, mais dont le bénéficiaire permanent est l'étymologie romane.

L'étymologie proprement française gagne beaucoup à être envisagée dans un ensemble intégrant, comme celui que von Wartburg a retenu pour le FEW. Il y a des solutions étymologiques qui resteront toujours hors de portée des étymologisations sectorisées ou bien qui resteront des options tentantes, mais impossibles à établir. Parmi les nombreuses propositions de Guiraud (1982), l'étymologie française a fait un tri sérieux. Il est notable que celle qu'il a proposée pour baliverner v.intr. 'tenir des propos futiles et creux' comme un sens métaphorique d'un verbe régional de l'ouest de la France *baliverner v.intr. 'marcher sans but, de façon erratique', composé des deux verbes du moyen français baller 'aller çà et là, chanceler' et *verner 'tourner', ait reçu le meilleur accueil. Cette reconstruction est évidemment très plausible. Elle repose sur un modèle possible, mais les étapes intermédiaires manquent, ce qui est un peu gênant pour une formation qui ne remonte qu'à la fin du Moyen Âge. Pour rencontrer le type intermédiaire il faut interroger des témoins que la lexicologie française évite généralement de fréquenter. On trouve un verbe balivèrnè v.intr. 'être sans cesse par voie et par chemin' dans un dictionnaire composé vers 1935 du patois poitevin d'un hameau de 132 habitants et c'est le seul glossaire dialectal de France qui, apparemment, le connaisse. Mais le Dictionnaire étymologique des créoles français de l'Océan Indien (DECOI) a répertorié sous le verbe baliverner, à côté du sens de 'plaisanter' qui est celui du français commun, des attestations réunionnaises aux sens de 'aller et venir', 'lambiner', 'errer' et mauricienne au sens de 'se promener en vue de se divertir' qui correspondent elles aussi au protosémantisme supposé par Guiraud. Il est exclu que ces trois données convergentes, recueillies dans des systèmes linguistiques éloignés géographiquement et typologiquement se soient développées indépendamment. Il n'y a pas à douter qu'elles documentent le sens archaïque, partout ailleurs effacé par le sens métaphorique du français, mais qui était encore courant dans la zone de départ des francophones partis dans l'Océan indien.

Au-delà de l'ampleur des matériaux traités, c'est aussi leur nature qui peut être considérée de façon originale dans le FEW. L'article ATTĚGIA (Billy / Chambon / Chauveau 1996 in FEW 25, 695b-700b) a repris l'essentiel d'un article antérieur de Chambon / Billy 1990 qui plaidait pour « l'intégration résolue de la toponymie et de l'anthroponymie dans une lexicologie lato sensu dont elles seraient des parties 'obligatoires' »(Chambon / Billy 1990: 62). Quand l'occasion s'en présente, les données onomastiques sont, sinon traitées exhaustivement comme dans cet article, au moins convoquées dans l'analyse sur un même pied que les données lexicales. L'examen systématique, par exemple pour l'article BATĀRE, de toutes les attestations de baie en rapport avec le domaine maritime permet de faire un tri entre lexèmes et toponymes bien mieux fondé que la lexicographie ne l'avait fait antérieurement. On évite ainsi les fausses sémantisations des désignations qui ont obscurci l'histoire du vocable, pour n'accorder un sens assuré qu'aux seuls lexèmes. L'imbrication des données lexicales et toponymiques s'en trouve clarifiée et leurs relations se dessinent nettement. Une substantivation du participe passé du verbe baer v.intr. 'être ouvert' a été utilisée comme descriptif topographique: baée n.f. 'estuaire d'un fleuve'. Celui-ci est devenu le nom d'un port de la côte atlantique situé dans l'estuaire d'un fleuve côtier: La Baie. La notoriété du lieu, 
fréquenté par toutes les marines d'Europe venues y charger du sel, lui a valu de devenir le prototype d'un renfoncement de la côte où les bateaux trouvent un abri sûr, et donc le détoponymique baie 'petit golfe'. La prise en compte raisonnée des données onomastiques oblige à catégoriser précisément chaque attestation, et la contrainte se révèle là aussi éclairante.

La complexité accrue des articles impose, pour permettre une lecture aisée, que la structuration des articles soit la plus explicite possible. À l'imitation du LEI, les principales articulations sont affichées en tête d'article et la hiérarchisation alpha-numérique évite désormais l'ellipse. Ce n'est pas seulement une meilleure mise en vitrine. Selon la tradition du FEW, mais de façon systématisée, les subdivisions intérieures sont signalées expressis verbis, en ce qui concerne, sur le plan formel, le mode de formation des mots (conversions, dérivés, composés, croisements, redoublements, etc.) et des unités syntagmatiques (locutions, proverbes) et les unités de morphologie constructionnelle (identification des préfixes et suffixes par leur forme latine ou française), de même que les figures de langue (métaphore, métonymie, etc.) sur le plan du contenu. Le lecteur ne devrait plus s'interroger sur la pertinence de tel ou tel classement, sur la place d'une donnée dans telle ou telle subdivision. Plutôt que de regrouper tacitement des matériaux disparates, il semble préférable de manifester le caractère énigmatique d'une formation, d'un sens spécifique ou l'appartenance douteuse d'une donnée à la famille lexicale traitée. Il est plus profitable de mettre en lumière les questions pendantes que de les escamoter. La justification explicite des classements des différents types morphologiques ou sémantiques proposés à l'intérieur de chaque article a également pour avantage d'obliger à une plus grande rigueur dans la catégorisation et permet d'aboutir à des explications mieux appropriées. Il n'est pas seulement question d'assembler ce qui se ressemble, mais de caractériser avec précision des types lexicaux à partir de toutes leurs attestations et de les enchaîner selon leur mode de formation. Ainsi la chronologie des attestations du substantif masculin français badaudage, dont les premières précèdent d'un siècle le verbe badauder, exclut d'en faire un dérivé exclusif de ce dernier. L'examen des données historiques impose de les scinder en deux types, les plus anciennes comme un dérivé du substantif badaud et les plus récentes comme un dérivé du verbe badauder, dérivé luimême tardif de badaud. On a donc affaire à deux homonymes diachroniques ( $c f$. Andronache 2009). Cette séparation oblige à reconsidérer les attestations les plus anciennes et à s'apercevoir que le vocable n'y a pas le sens du nom d'action moderne 'fait de se comporter comme un badaud', mais le sens d'un nom d'état: 'appartenance à la catégorie des badauds et spécifiquements des badauds de Paris, des habitants de Paris', conformément à sa dérivation à partir d'un substantif: le premier badaudage est une satire du parisianisme, des mœurs des habitants du Badaudois ou de La Badaudière, les surnoms moqueurs de la capitale jusqu'au début du $20^{\mathrm{e}}$ siècle. C'est l'analyse lexicologique qui fait comprendre les textes, et l'étude philologique n'est pas seulement une lecture syntagmatique. La commodité de la lecture implique la clarté de l'analyse. La hausse des exigences a un coût, mais aussi un intérêt.

Comme on le sait, l'établissement philologique rigoureux des données n'était pas considéré comme l'une des tâches essentielles des premiers volumes du FEW. La refonte en a fait une de ses priorités ( $c f$. Chambon 1989). L'aspect le plus spectaculaire de cette attention philologique, c'est la détection des pseudo-lexèmes auxquels la lexicographie historique accorde une existence fictive. La famille lexicale BĀJULA, BĀJULUS et BĀJULĀRE a été l'occasion de repérer neuf articles de cette nature dans les dictionnaires consacrés à l'ancien et au moyen français, qui sont désormais signalés dans la Base des motsfantômes (Steinfeld 2007-). Ces fantômes sont le plus souvent des égarés sans conséquence. Mais l'un deux, le pseudo ancien français *bail 'poutre', connaît une existence ininterrompue depuis cent ans dans les dictionnaires étymologiques romans ou de l'italien comme étymon de l'italien baglio 
n.m. 'trave ricurva disposta trasversalmente nello scafo, con funzione di sostegno e collegamento', depuis le REW ${ }^{1}$ jusqu'au DELI ${ }^{2}$ ( $c f$. BĀJULUS note 51 et Chauveau 2007).

L'une des tâches majeures de la refonte des étymons en B- du FEW est de réévaluer les propositions originales de von Wartburg dans la première rédaction. La refonte de l'article BĀSIOLUM rédigée par Christel Nissille (http://stella.atilf.fr/few/basiolum.pdf.) l'illustre au mieux. Cet étymon n'a connu aucun succès et il a été rejeté explicitement ou tacitement par les romanistes de renom comme Gamillscheg et Meyer-Lübke. Comme les correspondants évidents à première vue (de type fr. ‘baiseul ' 'baiser; baisure du pain') de cet étymon latin manquent aussi bien aux dictionnaires canoniques des états anciens des langues traitées qu'à ceux du français moderne et contemporain et ne se rencontrent que dans les parlers dialectaux de trois domaines linguistiques galloromans: français, francoprovençal et occitan, et eux seuls, ces matériaux n'ont aucune chance d'être étudiés, dans la perspective historique des études tant françaises que romanes, en dehors du cadre du FEW. La lexicologie historique centrée sur l'écrit ou sur la comparaison des langues romanes majeures n'est pas confrontée à un tel étymon, qu'elle peut écarter sans risque, de son point de vue sectoriel, et par làmême elle en perd les potentialités éventuelles. Car les données collectées et établies par la lexicographie moderne et contemporaine et leur insertion dans une reconstruction historique non seulement ne remettent pas en question l'hypothèse de von Wartburg, mais permettent d'en tirer une solution innovante: l'étymologie, jusque là disputée et irrésolue, de moyen français bisel 'facette d'un diamant', qui est à la source de français biseau 'bord taillé obliquement', mais aussi d'anglais bezel 'facette d'un diamant', d'occitan bisèu 'biseau', espagnol et portugais bisel et catalan bisell, tous empruntés au français. La comparaison interne au français (baisure, baiser du mitron), de même que romane (it. bacio del pane, toscan baciatura, catalan besada, besadura), montre que la liaison entre le baiser et un défaut de la croûte du pain est un fait largement partagé qui justifie de lier les dénominations de ce défaut: fr. $\ulcorner$ baiseul $\urcorner$, frpr. $\ulcorner$ beisòu $\urcorner$, occit. $\ulcorner$ baiòu $\urcorner$, à une dénomination du baiser. Il est également facile de relier à ce défaut, par une métonymie, les attestations anciennes de biseau qui signifient 'retaille de pain', pratiquée évidemment à l'endroit de ce défaut, avant de signifier, par une métaphore, 'facette plane d'un diamant obtenue par la taille'. Les difficultés d'ordre formel se résolvent facilement. Pour la finale, un article antérieur (Chauveau 2005) a mis au jour une petite liste d'étymons en -IOLUM dont le représentant est ou a été en -eau (RĒTIOLUM et réseau; *SCŪRIOLUS et afr. escureau; *AXIOLUM et afr. esseau), ce qui est également valable pour des toponymes (*MONASTERIOLU et Montereau; *PALATIOLU et Palaiseau; *PUTEOLU et Puiseau). Le radical bis- par rapport à bais- est bien connu, mais seulement à l'époque contemporaine. Le recours à l'onomastique (Wirth 2007 s.v. Baizatte) permet d'antédater celui-ci de cinq siècles. Ces deux ordres de faits autorisent à relier formellement biseau et baiseul, dont les liens sémantiques sont clairs par ailleurs.

La refonte du premier volume du FEW se focalise sur ce qui fait l'originalité méthodologique de l'ouvrage depuis ses débuts et cherche à l'amplifier. Elle a l'ambition de fournir une synthèse galloromane de l'histoire lexicale, en liaison constante avec les autres domaines romans. Cette synthèse est conçue comme englobant toutes les réalisations, hors de toutes limitations sectorielles et en mettant en relation le lexique galloroman avec ses expansions hors de son berceau et avec ses parallèles onomastiques. Elle vise à fournir la description la mieux informée et l'explication la plus précise de chacune des articulations qu'elle est amenée à distinguer dans la variation lexicale envisagée sous tous les angles qu'elle connaît. Elle met en œuvre pour y parvenir l'information lexicale la plus étendue, traitée avec un souci philologique accru et analysée avec toutes les ressources de l'analyse linguistique diachronique. La contrepartie en est que la visée totalisante du thesaurus galloromanicus poussée à son maximum a dû se concentrer sur quelques segments réunis dans un champ 
d'expérimentation où les méthodes étymologiques peuvent prouver leur efficacité. L'ambition de la somme étymologique ne se conçoit plus pour l'instant dans un seul ouvrage, mais comme la cumulation d'entreprises sectorielles supposées se compléter les unes les autres.

\subsection{DEAF}

Le DEAF se présente lui-même comme un «petit FEW pour le domaine et l'époque de l'ancien français » (Baldinger 1971 in DEAF G 1 XIII; cf. Möhren 2003 pour une présentation plus récente), c'est-à-dire pour la période s'étendant de 842 au milieu du $14^{\mathrm{e}}$ siècle. En réalité, ce dictionnaire, dirigé, depuis le départ à la retraite officiel de Frankwalt Möhren en août 2007, par Thomas Städtler, est bien plus que cela. De von Wartburg, il a repris la conception de l'étymologie-histoire, qui se révèle dans sa macrostructure (regroupement du lexique de l'ancien français par familles étymologiques) et dans la microstructure des articles (principe de classification par dépendance génétique des formes et des sens et discussion étymologique détaillée et critique). Très rapidement toutefois (dès le deuxième fascicule, paru en 1973), les principes philologiques rigoureux qui président à la rédaction des articles l'ont conduit à se démarquer de son modèle sur divers points, dans le souci notamment d'offrir une analyse sémasiologique complète des unités décrites. Contrairement au FEW, le DEAF sépare le système graphique du système sémantique, il veille à ne pas disperser les divers sens d'un lexème et il complète la définition par des citations à portée encyclopédique. Le retour systématique aux sources et l'identification minutieuse des textes cités ailleurs (notamment par Gdf et TL) sous des sigles variés ont en outre donné naissance à une bibliographie (DEAFBibl, dont la version électronique, DEAFBiblEl, tenue à jour, est consultable sur Internet) qui s'est imposée très rapidement comme une référence en la matière. Avec son complément bibliographique, ses index et depuis peu sa version électronique (DEAFEl: DEAFplus [DEAF papier en mode image] et DEAFpré [matériaux lemmatisés]), le DEAF n'offre pas seulement un examen plus détaillé et plus complet d'un secteur diachronique couvert par le FEW, mais représente un outil proprement irremplaçable pour la linguistique et la philologie de l'ancien français.

Si le DEAF constitue le dictionnaire de référence de l'ancien français, son envergure ne se limite nullement à cet état de langue: les recherches réalisées en son sein sont pertinentes pour l'histoire du lexique français, galloroman, voire roman en général. Un sondage à partir des 35 premières pages du fascicule $\mathrm{J} 4-5 / \mathrm{K}$ (2008, colonnes 573-642) met ainsi en évidence les apports suivants au FEW:

- deux nouveaux étymons: JUVENCA, à insérer FEW 5, 92a (Dörr in DEAF J 634 s.v. *jovence) et persan HiRDAMYĀN, à insérer FEW 19, 66b (Möhren in DEAF J 589-590 s.v. jordanian);

- un étymon à supprimer: mnéerl. *JOFFROU (von Wartburg 1956 in FEW 16, 284b), article dont la seule donnée est à transférer s.v. JŬGUM (FEW 5, 61a), de même que ses congénères classés par von Wartburg 1968 in FEW 23, 97a, 'agrès' (Dörr in DEAF J 633 s.v. jouvre);

- la mise en évidence du triple classement (sans renvois réciproques) de jorroise n.f. 'sorte de prune': (1) von Wartburg 1948 in FEW 5, 51a, JOUARRE, (2) Hoffert / Hubschmid / Lüdtke 1965 in FEW 21, 74b, 'fruit' et (3) Hubschmid 1965 in FEW 21, 84b, 'prunier; prune' (Tittel in DEAF J 591 s.v. jorroise);

- une proposition de correction étymologique: "Les <dérivés> réunis avec joscle dans le FEW sont p.-ê. partiellement à joindre à $\rightarrow$ JOU (p. ex. son $<$ frcomt.> joclé 'attaché au joug', Du Pinet éd. 1566) » (Möhren in DEAF J 592 s.v. joscle);

- la dénonciation d'un mot fantôme: achamp. josner v. 'repaître' (Hoffert / Hubschmid / Lüdtke 1969 in FEW 21, 455b, 'nourrir', Dörr in DEAF J 593 s.v. *josner; cf. aussi Dörr in DEAF J 597 s.v.joster pour un mot fantôme dans Gdf [justeür]); 
- des corrections de descriptions sémantiques: « le FEW fragmente la signification de jote en considérant les différentes sortes de légumes utilisés pour la préparation, suivant les déf. des dictionnaires et glossaires. Pour les att. afr. et mfr., cela ne convainc pas, car le genre du légume employé ne fournit pas de sème distinctif (de plus, il y est souvent impossible de savoir de quel légume il s'agit)" (Tittel in DEAF J 621 s.v. jote); «TL 4,1820 donne une définition 'weltliches Treiben' [...]. Or, un tel sens n'existe pas [...] » (Dörr in DEAF J 641 s.v. jovent).

En outre, ces mêmes pages fournissent quatre antédatations par rapport à l'information disponible dans le TLF: joute $\left(1178 \rightarrow 1^{\text {ère }}\right.$ moitié du $12^{\mathrm{e}}$ siècle, Dörr in DEAF J 603 s.v. joste), jouvenceau ( $1^{\text {ère }}$ moitié du $12^{\mathrm{e}}$ siècle $\rightarrow 1^{\mathrm{er}}$ quart du $12^{\mathrm{e}}$ siècle, Dörr in DEAF J 637 s.v. jovencel $)$, rajouter $\left(15^{\mathrm{e}}\right.$ siècle $\rightarrow 3^{\mathrm{e}}$ quart du $12^{\mathrm{e}}$ siècle, Dörr in DEAF J 617 s.v. rajoster $)$ et surajouter $\left(1314 \rightarrow 1^{\mathrm{er}}\right.$ quart du $13^{\mathrm{e}}$ siècle, Dörr in DEAF J 618 s.v. sorajoster $)$.

Dans le même ordre d'idées, on citera le va-et-vient fructueux entre description lexicographique et analyse lexicologique qui est consubstantiel à toute recherche menée par l'équipe de Heidelberg, mais qui se manifeste avec une clarté particulière quand le DEAF se met au service de collaborations externes. Ainsi l'étude de la pragmaticalisation progressive de l'adverbe toujours menée par l'une d'entre nous (Buchi 2007) a grandement profité de l'apport de Thomas Städtler, dont les dépouillements ciblés ont permis d'étayer la filiation postulée entre l'emploi persistant ('encore au moment considéré') et l'emploi scalaire ('au moins, quoi qu'il en soit par ailleurs [affirme une validité résistant à toute éventualité]', ainsi dans C'est toujours ça!) par la preuve de l'existence de toujours persistant dès la période de l'ancien français. Inversement, le lexicographe n'a pas hésité à faire fructifier l'article en question pour le DEAF: « la structuration sémantique que nous suivons ici est celle proposée par Éva Buchi, <Sur la trace de la pragmaticalisation de l'adverbe toujours $>$, à paraître » (Städtler 2006 in DEAF J 564 s.v. toz jors).

Un bel exemple de potentialisation réciproque est fourni de même par l'article */' $\beta \mathrm{ad}-\mathrm{u} / \mathrm{du}$ DÉRom, rédigé par Julia Alletsgruber, qui s'appuie sur la modélisation unifiée fournie par Möhren 2000 sur la base de l'analyse de 54 lexèmes en $g u$ - traités par le DEAF pour récuser toute influence germanique dans la genèse de fr. gué et de ses congénères, et qui permet en même temps, à partir de l'aréologie des issues de */'uad-u/, qui assigne ce dernier à un stade plus récent du protoroman que */' $\beta \mathrm{ad}-\mathrm{u} /$, de rejeter l'hypothèse d'un conservatisme telle qu'elle est proposée par Möhren (2000: 51, 54-55, 62) et par là d'ouvrir la voie à d'autres hypothèses interprétatives (hypercorrectisme ?).

\subsection{TLF-Étym}

En 2004, l'ATILF a doté le TLF d'une version informatisée (TLFi). Ce nouveau format facilite non seulement la consultation des matériaux du dictionnaire, mais aussi leur mise à jour. C'est dans cet objectif qu'a été lancé en janvier 2005 le programme de recherche TLFÉtym, une révision sélective des notices étymologiques du dictionnaire (cf. Buchi 2005). On sait que le TLF se situe à la charnière de la recherche scientifique et de la vulgarisation. Ses rubriques étymologiques, qui proviennent d'une utilisation critique des matériaux du FEW, constituent la source principale (sinon unique) des dictionnaires de vulgarisation en lexicographie française historique. Le Robert historique a par exemple largement puisé dans le TLF, souvent sans vérification aucune des données (cf. Thomassen 1997). Or, ces dernières sont sujettes à de nombreuses corrections.

Le programme de recherche TLF-Étym, dirigé par Nadine Steinfeld et bénéficiant de l'apport de Frankwalt Möhren, ancien directeur du DEAF, comme consultant scientifique, se nourrit des résultats des recherches menées par l'équipe «Linguistique historique française et romane » de l'ATILF, à laquelle appartiennent notamment le FEW et le DMF. Il bénéficie 
également de la coopération ponctuelle ou régulière de chercheurs français et étrangers et accepte les propositions de corrections soumises par quiconque via un formulaire. Des méthodes de travail rigoureuses (méthode historico-comparative, analyse philologique des textes anciens, métalexicographie et critique des sources) permettent d'apporter de nombreuses corrections, au niveau tant des étymologies que des attestations fournies.

Par ailleurs, TLF-Étym a représenté la structure d'accueil naturelle pour les étymologies revues dans le cadre du projet ANR DETCOL (Développement et Exploitation Textuelle d'un Corpus d'Euvres Linguistiques), dirigé par Bernard Colombat (HTL, CNRS \& Université de Paris 7) et développé durant les années 2007-2011. La refonte d'une centaine de notices étymologiques du TLFi dans ce cadre a ainsi fait dégager de nouvelles étymologies pour archaïsme (emprunt au grec), cognitif (emprunt à l'anglais), corpus (emprunt à l'allemand), dialectologie (emprunt à l'allemand), gémination (emprunt au latin et dérivation interne), gérondif (emprunt au latin), hypercorrection (emprunt à l'anglais), imparfait (ellipse de prétérit imparfait), morphologie (emprunt à l'allemand), néologique (dérivé de néologie), néologisme (dérivé de néologie), néologiste (dérivé de néologisme), oraison (lexème héréditaire), parfait (ellipse de prétérit parfait), passé (ellipse de temps passé), patronymique (emprunt au latin), phonologie (emprunt à l'anglais), plus-que-parfait (ellipse de prétérit plusque-parfait), pragmatique (emprunt à l'anglais), psycholinguistique (emprunt à l'anglais), ruthène (emprunt au latin ecclésiastique), sémantique (emprunt au grec), subordonné (transcatégorisation à partir du participe passé du verbe subordonner) et temporel (emprunt au latin), ainsi que des dizaines de corrections de dates (notamment des antédatations, mais aussi quelques rétrodatations, particulièrement précieuses).

Au-delà de ces apports concrets, l'importance stratégique du programme TLF-Étym réside surtout dans l'importante réflexion méthodologique qu'il a générée dès son lancement, que ce soit à travers la journée d'étude « Lexicographie historique française: autour de la mise à jour des notices étymologiques du Trésor de la langue française informatisé », qui s'est tenue en novembre 2005 à l'ATILF (cf. Buchi 2006/2007), le séminaire de méthodologie en étymologie et histoire du lexique (année universitaire 2005 / 2006, cf. Buchi 2005-2007) ou dans des domaines particuliers comme la continuité lexicale (Andronache 2009), les « faux amis » intralinguistiques (Steinfeld 2009), les voies d'emprunt au latin (Steinfeld / Andronache 2011) ou encore les déonomastiques (Leroy 2009).

\section{Lexicographie spécialisée}

\subsection{Ensembles étymologiques}

Le lexique héréditaire français ne bénéficie pas d'un dictionnaire dédié, mais il est traité, au même titre que celui des autres langues romanes, dans le Dictionnaire Étymologique Roman (DÉRom). Ce dictionnaire se singularise fortement dans le paysage de l'étymologie romane par son orientation méthodologique vers la grammaire comparée-reconstruction. Il s'agit là du paradigme hégémonique en étymologie héréditaire de toutes les familles linguistiques du monde, à l'exception notable de l'étymologie romane, qui estime généralement pouvoir s'en passer avec profit en raison du témoignage massif des données du latin écrit de l'Antiquité. En reconstruisant leurs étymons à partir de séries de cognats romans, unités lexicales apparentées entre elles en vertu de leur héritage commun du protoroman, les rédacteurs du DÉRom s'emploient ainsi à rapprocher l'étymologie romane de l'étymologie celtique, germanique, slave, indo-européenne, bantoue, austronésienne etc., et par là à intégrer l'étymologie romane à l'étymologie générale. Un article récent (Buchi et al. 2010) explore les implications théoriques du paradigme proposé par le DÉRom pour l'étymologie française, mais ce dernier n'a pour l'instant guère été appliqué en lexicographie historique française. 
Le lexique de formation française - qu'il s'agisse de créations, par dérivation, composition etc., de nouveaux vocables ou de dégagements, par métonymie, métaphore etc., de nouveaux lexèmes se rattachant à des vocables déjà existants - ne dispose pas non plus d'un dictionnaire qui lui serait consacré. En revanche, les résultats des recherches portant sur l'emprunt linguistique se présentent volontiers sous une forme lexicographique. Pour le domaine français et galloroman, l'ouvrage le plus important d'un point de vue méthodologique porte sur les emprunts aux langues orientales (Arveiller 1999). Une quatrième «classe » étymologique est constitué par le lexique d'origine inconnue, qui forme la matière des Etymologien de Baldinger (Baldinger 1988-2003). Ces deux sommes de travaux ont été conçues, dès le départ, comme des compléments correctifs du FEW.

\subsubsection{Les Orientalia d'Arveiller}

La monographie d'Arveiller (1999) est un recueil posthume des 25 articles que l'auteur avait publiés dans la Zeitschrift für romanische Philologie, entre 1969 et 1996, sous le titre de " Addenda au FEW XIX ». Ce titre correspond très bien au contenu de l'ouvrage, qui reprend en quelque six cents pages, et en suivant l'ordre alphabétique des étymons retenu par von Wartburg, quelque 270 des articles de ce volume du FEW pour y apporter des compléments de diverses natures. L'introduction du premier article indique qu'il ne faut pas s'attendre à un « examen critique de cet excellent fascicule » (11), mais, comme von Wartburg lui-même y invitait ses lecteurs, à des précisions sur les datations, à des éclaircissements sur les « cheminements de langue à langue » et à des contributions à la détermination de quelques « origines ». Le travail se donne comme un commentaire érudit du volume du FEW consacré aux emprunts lexicaux du galloroman aux langues du Proche-Orient.

En fait, ce qui fait l'originalité et l'intérêt de l'ouvrage, c'est qu'il est bâti sur un matériel lexical entièrement nouveau par rapport à celui dont disposait von Wartburg, obtenu par la lecture et le dépouillement systématique de quelques centaines de sources relatives au ProcheOrient. Les articles du FEW qui sont examinés le sont à la lumière d'une masse impressionnante d'attestations nouvelles qui conduisent souvent à réinterpréter les données antérieures et à modifier l'histoire de ces emprunts. Cela permet d'établir, par exemple, que les plus anciennes attestations que l'on donne de ramadan, emprunt à l'arabe, et ramazan, emprunt au turc, sont à situer dans une perspective à long terme. La première se rencontre, isolément au $15^{\mathrm{e}}$ siècle, dans une traduction de l'italien et bon nombre des mentions ultérieures donnent le lexème en citation, comme un mot de l'arabe ou du turc. Aux $16^{\mathrm{e}}$ et $17^{\mathrm{e}}$ siècles, ramadan oscille donc encore entre xénisme et " début d'emprunt » (437). L'histoire lexicale devient moins schématique et plus proche de la réalité linguistique. Les intermédiaires entre le français et les langues du Proche-Orient sont déterminés avec plus de justesse. L'italien est l'intermédiaire entre le turc QAFȚĀN et mfr. frm. cafetan au $16^{\mathrm{e}}$ siècle (225-227), dont la vitalité a été assurée ultérieurement par des contacts directs. Semblablement l'emprunt à l'arabe ḤAŠǏš passe d'abord par l'italien au $16^{\mathrm{e}}$ siècle, d'où hasis, puis par le latin scientifique assis aux $17^{\mathrm{e}}$ et $18^{\mathrm{e}}$ siècles, ensuite par l'allemand Haschisch, enfin c'est seulement au début du $19^{\mathrm{e}}$ siècle que s'établit le contact direct des francophones avec l'arabe en Égypte et au Maghreb, d'où hachich: la variation graphique du mot en français contemporain est tributaire de cette histoire (184-190). Dans l'histoire de frm. marabout (397-400) on fait intervenir l'arabe MURĀBIṬ, mais aussi sa réalisation dans l'arabe du Maghreb, où il est "prononcé à peu près merābot» (399), de même que l'emprunt espagnol morabito et le portugais maraboto / marabuto. Ce qui permet de faire le partage, ce sont les contextes dans lesquels apparaît l'emprunt et l'examen des contacts linguistiques prévalents. On peut ainsi exclure le portugais du processus et traiter comme un emprunt à l'espagnol les formes de type morabite, attestées entre 1613 et 1748 , tandis que «le type 
marabou(t) qui se lit d'abord dans des récits d'aventures au Maghreb » (399) doit être un emprunt à l'arabe de cette région. Pour déterminer avec assurance ce qui, dans les dénominations du café, remonte au turc QAHVE ou à l'arabe QAHWA (229-245), il faut se livrer à une minutieuse analyse comparative des graphies anciennes, avec recours au manuscrit quand c'est possible, pour déterminer la nature de la consonne intérieure représentée par des graphies $<\mathrm{u}, \mathrm{vv}, \mathrm{uu}, \mathrm{o}, \mathrm{ou}, \mathrm{oü}>$, seule marque susceptible de distinguer ce qui appartient au type de frm. café, emprunté au turc, et ce qui relève du type de frm. caoua, emprunté à l'arabe. Ce dernier se révèle ainsi plus ancien de deux siècles qu'on ne croyait. L'examen, tant formel que sémantique, des données mises en rapport pour expliquer $\mathrm{mfr}$. frm. caffarre / caphar 'sorte de tribut que les Turcs exigeaient des pèlerins chrétiens' (217-222) permet de réfuter l'étymon KAFFĀRA 'sacrifice expiatoire' au profit de HAFĀRAH 'action d'escorter qn pour le protéger'.

L'étymologie-histoire que von Wartburg voulait mettre en œuvre dans le FEW connaît ainsi un nouveau développement. Les orientalismes, tels qu'ils étaient traités dans la lexicographie historique dont disposait von Wartburg, fournissaient un matériel très imparfait pour l'histoire qu'il voulait écrire. Les nombreux matériaux qu'Arveiller a su réunir lui ont donné la base nécessaire pour éclairer de manière plus précise, et assez souvent décisive, les contacts linguistiques entre le français et les langues du pourtour méditerranéen qui ont laissé une trace dans le lexique galloroman. On aboutit par cette voie à un traitement comparable pour le lexique emprunté à celui qui a été donné au lexique héréditaire.

\subsubsection{Les Etymologien de Baldinger}

Dès avant la fin de la parution des volumes 21 à 23 du FEW, qui rassemblent le lexique galloroman dont l'étymologie n'est pas assurée ou bien est inconnue, Kurt Baldinger avait commencé à explorer ces matériaux, que von Wartburg lui-même jugeait «les plus intéressants, ceux, en tout cas, qui animeraient le plus la discussion » (Wartburg 1953: 113). Il avait proposé à ses étudiants de Heidelberg et de Strasbourg de s'exercer dans ce « magnifique terrain de jeux étymologiques» (Baldinger 1974: 43). Une bonne vingtaine d'années plus tard, les trois volumes parus des Etymologien réunissaient l'ensemble des contributions, sur plus de 1500 pages, de Baldinger, de ses élèves, de ses collaborateurs et de ses correspondants, au total une soixantaine de personnes. En outre avaient été dépouillés les publications suscitées antérieurement par ces mêmes "inconnus", comme les travaux lexicologiques qui avaient traité de tel ou tel de ces matériaux.

L'ouvrage n'est pas conçu comme une réélaboration des trois volumes dits des « inconnus » du FEW, ni même comme une révision étymologique, mais comme la collection la plus large possible des corrections apportées par un ensemble de romanistes aux intérêts spécifiques. C'est ainsi que le lexique de l'ancien français a bénéficié des révisions des collaborateurs du DEAF (Stephen Dörr; Frankwalt Möhren; Thomas Städtler), que celui du wallon a été l'objet de nombreuses rectifications de la part des éditeurs de l'Atlas Linguistique de la Wallonie (Marie-Guy Boutier; Marie-Thérèse Counet; Jean Lechanteur), que le vocabulaire de la marine a été repris par Jan Fennis en marge de ses travaux sur ce secteur lexical, que d'autres proposaient des élargissements aux emprunts à l'arabe ou de nouveaux arabismes (MartinDietrich Glessgen; Norbert Weinhold), des emprunts au grec (Norbert Wienhold) ou encore au malgache (Tiana Shabafrouz).

L'ouvrage est un indispensable complément du FEW, dont il renforce la cohérence. La plupart des notices se consacrent à rapatrier dans leur famille des données simplement égarées comme frm. barouf $\left(\mathrm{n}^{\mathrm{o}} 3800\right)$ ou frm. bouquinquan $\left(\mathrm{n}^{\mathrm{o}}\right.$ 1894), en y précisant à l'occasion l'histoire du mot, comme pour la locution frm. de bric et de broc ( $\left.{ }^{\circ} 3845\right)$. Beaucoup de notices procèdent à l'identification des types lexicaux qui se cachent sous une forme insolite, 
comme frm. barras, qui est une variante de borax $\left(\mathrm{n}^{\circ} 188\right)$, ou mfr. béturre, qui est une variante suffixale de frm. bétoire $\left(\mathrm{n}^{\circ}\right.$ 5657). L'une des étapes de la recherche étymologique consiste à reconstituer les liens historiques et génétiques qui rapprochent des types lexicaux distincts, mais dont la paronymie et la parasynonymie demandent à être examinées, comme ceux qui peuvent être établis entre les termes argotiques ou d'origine argotique bistro, bistingo, bastringue, mastroquet $\left(\mathrm{n}^{\circ}\right.$ 2595). Certaines notices font simplement le point sur les diverses propositions qui ont été faites depuis la publication de ces « inconnus », comme pour frm. bécane ( $\mathrm{n}^{\circ}$ 3247), bigoudi ( $\mathrm{n}^{\circ}$ 1989, 5270), boui-boui $\left(\mathrm{n}^{\mathrm{o}} 2743,3645\right)$ ou encore cambouis $\left(\mathrm{n}^{\mathrm{o}}\right.$ 3190). Au total, c'est surtout la lexicographie dialectale ou du français populaire, celle qui avait été la moins travaillée avant l'œuvre de von Wartburg et pour laquelle il pouvait le moins s'appuyer sur des travaux préparatoires, qui est la plus présente dans les Etymologien et qui en tire le plus de bénéfices. Au bout du compte, les Etymologien confortent l'originalité de l'objectif de von Wartburg qui visait à traiter globalement, sur le même plan et selon les mêmes méthodes, les diverses instanciations du lexique galloroman.

Il est, d'autre part, notable que sur les quelque huit cents renvois à un étymon à initiale $b$-, seule une quarantaine correspond à l'un des trente-trois étymons qui sont nouveaux par rapport à ceux qui forment la nomenclature du FEW. Parmi ces derniers, les nouveaux étymons latins ont, comme on s'y attend, la part la plus congrue: seuls BĪDUĀNUS $\left(\mathrm{n}^{\circ} 3484\right.$, Baldinger) et *BULLīTELLUS ( $\mathrm{n}^{\circ}$ 91, Renard). Les langues de substrat, identifiées ou non, fournissent le plus gros contingent: gaul. *BAGARETO- $\left(\mathrm{n}^{\circ}\right.$ 950, Hubschmied), *BOU- $\left(\mathrm{n}^{\circ} 549\right.$, Hubschmid), *BREGETO- $\left(\mathrm{n}^{\circ}\right.$ 4682, Hubschmied), *BRŪSCA ( ${ }^{\circ}$ 5453, Desponds), *BRUSCIA (n ${ }^{0}$ 280, Desponds), *BRŪTO- ( $n^{0} 377$, Hubschmied); prélat. *BARRŪCA ( $n^{\circ} 393$, Bernhard), *BIKKO- (n $\mathrm{n}^{\mathrm{0}}$ 1386, 2592, 2606, 3946, 5817, Hubschmid), *BŪC- $\left(\mathrm{n}^{\circ}\right.$ 66, 83, 3720, 3728, 4051, Aebischer / Schüle), *BŪLI- $\left(n^{0} 3479\right.$, Keller / Jeanjaquet); indo-européen *BRUGHNO- $\left(n^{0} 373\right.$, Hubschmid); préindo-européen *BAKASTO- / BAKATTO- ( ${ }^{0} 3647$, Hubschmid). Les emprunts anciens aux langues germaniques ne sont évoqués que par ancien norrois BAITI-ÁSS ( ${ }^{\circ} 3327$, Fennis) et francique BALTI $\left(\mathrm{n}^{\mathrm{o}} 2759,3589\right.$, Gamillscheg), BOL- (287, $\mathrm{n}^{\circ}$ Weinhold). Les langues modernes sont représentées dans ces nouveaux étymons par arabe BARǴAŠ $\left(n^{0} 766\right.$, Weinhold), BAȚT,A ( ${ }^{0}$ 4015, Weinhold), BIS'-SIF ( $n^{\circ} 3974$, Esnault), breton BOAN ( $n^{0} 4233$, Chauveau), hindi BA(I)YA ( ${ }^{\circ}$ 631, Weinhold), moyen néerlandais BU(Y)SE $\left(n^{\circ} 65,4050\right.$, Baldinger), romani BENG ( $\mathrm{n}^{\circ} 3588$, Baldinger), turc BINDALLI $\left(\mathrm{n}^{\circ} 1975\right.$, Weinhold). Enfin ont été repérées quelques formations internes, à partir de données onomastiques comme BANBAN NP ( $n^{\circ}$ 3009, Herbillon/Legros), BASIN NP ( $n^{\circ}$ 2, Legros), BASTOGNE NL ( $n^{0}$ 5129, Lechanteur), BASTOS NP ( $n^{\circ} 3613$, Esnault), BOULIÈNE NL ( ${ }^{\circ}$ 336, Legros), BOURGOIN NL ( $\mathrm{n}^{\mathrm{o}}$ 5033, Chambon), ou à partir d'onomatopées comme BLOUM ( ${ }^{\circ}$ 3600, Baldinger), BÔM' ( $\mathrm{n}^{\mathrm{O}}$ 5929, Counet). Même si tel ou tel de ces étymons a pu ou pourra être contesté, la plupart d'entre eux seront retenus. Les innovations sont significatives et dessinent par contraste les domaines les moins aboutis du FEW de von Wartburg: le substrat, les contacts linguistiques modernes et les relations avec l'onomastique. Les Etymologien ont apporté dans ces différentes directions des progrès sensibles qui améliorent la couverture étymologique du lexique galloroman fournie par le FEW. Que ce soit par le débuscage des "faux inconnus », par la mise au point de l'état de la recherche ou par la détermination de nouveaux étymons, l'ensemble des Etymologien constitue ainsi le plus important prolongement du FEW de von Wartburg.

\subsection{Variétés diatopiques}

\subsubsection{Variétés dialectales}

La lexicologie des variétés diatopiques galloromanes n'avait pas attendu le FEW pour adopter une perspective étymologique, mais cette voie a été peu fréquentée dans le domaine français, 
à quelques exceptions d'autant plus remarquables. Ce n'est que tardivement, c'est-à-dire lorsque la majeure partie du FEW aura été disponible, que la lexicologie dialectale a jugé utile de situer étymologiquement ses collectes (Rézeau 1976) ou ses études lexicales (Lerond 1963; Lepelley 1974) par des références systématiques au FEW. Pour ce qui est des collectes cartographiées, deux entreprises se détachent: l'Atlas linguistique de la Wallonie (ALW) et l'Atlas linguistique de la Franche-Comté (ALFC), avec des procédures originales dès la conception. Tandis que l'atlantographie belge a conçu dès le départ la présentation de la collecte comme ordonnée par l'étymologie, son homologue française a rélégué l'étymologie dans les index terminaux.

Il est significatif que soit décrit comme "avant tout un index étymologique " (Dondaine 2002: IX) l'ouvrage qui recense d'un point de vue étymologique le matériel lexical recueilli par l'ALFC. Les types lexicaux y sont illustrés par un court extrait du passage du FEW où il est étudié, ainsi que d'une référence à l'entrée et à la notice étymologique correspondante du Glossaire des patois de la Suisse romande (GPSR), qui couvre une zone géographique contiguë et dont la partie méridionale appartient elle aussi au francoprovençal. Un bref commentaire accompagne ces références et argumente, s'il en est besoin, les solutions étymologiques retenues. Ces citations établissent la liaison entre la collecte et la lexicologie française et francoprovençale et les études étymologiques de référence. Leur importance peut s'évaluer au fait que l' « index des bases étymologiques » (Dondaine 2002: 563-580) comporte près de trois mille entrées. Un autre index recense une centaine de passages des " inconnus » du FEW pour lesquels des étymologies ont été proposées. Les doubles étymologisations du FEW sont signalées et corrigées. Complétés par Chambon (2003), ces différents index confirment, précisent, renouvellent ou rectifient l'histoire lexicale des parlers dialectaux d'une zone à cheval sur deux domaines linguistiques.

À la différence des atlas réalisés en France, l'ALW est, dans sa conception, un atlas interprétatif. Les données recueillies y sont présentées dans un tableau qui organise les différents types formels classés dans une perspective historique. Le regroupement des formes n'est pas fondé sur leur similitude mais sur une analyse historique qui, par exemple, conduit à diviser en deux catégories des homophones de même sens, tels que seûr / seur 'sœur', selon qu'ils représentent le résultat dialectal régulier, dans la partie picarde, ou bien un emprunt au français, dans le sud de la Wallonie (ALW 17, notice 40). Ce n'est pas seulement in fine, mais dès le classement des matériaux qu'est adoptée la perspective génétique. De ce fait la structuration des notices est très liée au FEW, non seulement pour les types étymologiques, mais jusque dans la structuration inférieure parfois: ainsi les dénominations de la source par des dérivés de SURGERE reprennent explicitement les divisions établies par von Wartburg selon la consonne finale du radical sorg-, sord- et sors- (ALW 6, 43). Chaque unité de structuration est commentée d'un point de vue étymologique dans des notes où le FEW est au minimum référencé, mais bien souvent complété, corrigé, ou réfuté selon qu'il y a lieu. De ce fait s'y développe une véritable réévaluation de l'état de l'art représenté par le FEW, en fonction des matériaux nouveaux et aussi en référence à des travaux lexicologiques récents ou non. Les doubles classements étymologiques sont signalés, les solutions du FEW parfois remises en cause. Des propositions plus économiques sont argumentées: par exemple, les deux prototypes *DERVA et *DERVULA distingués par le FEW ne sont pas nécessaires, car toutes les données peuvent représenter *DERVULA (ALW 8, 21); ou les deux types laye et naye 'limite d'une coupe de bois', en raison de leurs configurations spatiales, peuvent être rattachés à un même étymon ancien bas francique *LAIDA, sans qu'il soit besoin d'un étymon moyen néerlandais NAET (ALW 8, 512); une nouvelle donnée (ALW 8, 82) rend inutile l'emprunt médiéval de l'allemand HAG retenu par le FEW $(16,115 \mathrm{a})$. À l'inverse, de nouveaux étymons sont proposés comme le luxembourgeois KLÖPPEL (ALW 8, 487). 
Quel que soit l'endroit où ils fassent intervenir le FEW, ces travaux dialectaux ne se conçoivent pas sans lui, à partir du moment où ils adoptent une perspective étymologique. Ils $\mathrm{y}$ sont quasiment contraints par le fait que le FEW est le thesaurus de la lexicographie dialectale galloromane et qu'il en fournit une analyse étymologique englobante sans aucun équivalent. Tel n'est pas le cas pour les régionalismes du français, et pourtant la lexicologie régionaliste y recourt intensément.

\subsubsection{Variétés régionales}

Parmi les grands dictionnaires de régionalismes ce fut celui de l'équipe du Trésor de la langue française au Québec (cf. Poirier 1985) qui fut le premier mis en chantier, dans les années 1970. Les régionalismes étaient majoritairement à cette époque conçus comme des dialectalismes qui avaient connu un changement de statut ou de langue. L'absence du niveau dialectal au Canada francophone, la prise en compte très réduite alors des régionalismes dans la lexicologie diatopique et la médiocre utilisation des données canadiennes dans le FEW ne plaidaient pas pour l'adoption d'une perspective historique et étymologique. Pourtant l'entreprise fut bâtie dès le départ pour correspondre à ce qui deviendrait ultérieurement son titre: Dictionnaire historique du français québécois (Poirier 1998). Cette perspective allait à la rencontre des attentes du public: "l'orientation historique et étymologique du dictionnaire répond à un besoin profond de la société québécoise encore aux prises avec un difficile problème d'identité » (Poirier 1985: XIII). Dans une situation d'insécurité linguistique les preuves d'ancienneté et d'ancrage dans la langue avant l'émigration valent sinon brevets de noblesse, du moins certificats de légitimité. Le dictionnaire s'attache avant tout à décrire le vocabulaire québécois spécifique en l'illustrant par diverses citations puisées dans l'écrit littéraire ou documentaire et en mettant en évidence ses usages et leur répartition, mais chaque article comporte une notice historique. Les nécessités de la publication ont amené à réduire le volume de celle-ci par rapport aux premiers essais, mais elle répertorie de façon systématique la plus ancienne attestation, elle spécifie la voie par laquelle le mot est entré en français québécois ("héritage de France », " héritage des parlers de France », " emprunt de », "par spécialisation », " dérivé de », " forme atténuée de » etc.) et elle détermine son étymon. En tant que variété de français, c'est beaucoup moins l'étymon lointain que l'étymon proche qui lui importe. Les notices vont prendre de l'importance lorsque le FEW (11, 314, SCIŪRUS) n'a pas distingué les types écureuil, écureux et écureau, entre lesquels le français québécois et le français d'Europe ont fait des choix différents ou bien lorsque le FEW n'a que peu ou pas de données (FEW 6/3, 140b, MORPHE), par exemple, pour les variantes amorphoser / emmorphoser / morphoser 'métamorphoser' dont la documentation nouvelle révèle la présence dans les créoles et chez les conteurs traditionnels de l'ouest de la France, ou bien lorsque le FEW est muet (FEW 20, 18b, OREIN) sur les rapports entre les formes orignac, orignat, orignal, original, ou encore lorsque l'histoire du mot est propre au Québec, comme traversier face à ferry-boat en France. C'est l'étymologie-histoire qui prime'

À la différence du précédent, le Dictionnaire Suisse Romand (DSR) comporte une « liste des étymons du FEW cités dans les commentaires » (789-800) accompagnés éventuellement des mentions « double classement», "faux classement», " étymon à ajouter», " étymologie controversée ». L'étymologie-origine n'est pas délaissée, mais elle reste secondaire par rapport à l'histoire lexicale. Du fait que dans la majeure partie du domaine couvert, la langue romane héréditaire est le francoprovençal et que le français s'y est introduit à partir du $13^{\mathrm{e}}$ siècle à l'écrit et qu'il y a concurrencé à l'oral le dialecte à partir du $17^{\mathrm{e}}$ siècle, la notice

\footnotetext{
${ }^{1}$ En complément et sur le même modèle a été composé un Dictionnaire du français acadien, qui fournit une notice historique pour les acadianismes du bon millier d'entrées traitées dans l'ouvrage qui ne sont pas usités au Québec (Cormier 1999).
} 
historique doit établir comment chacune des unités est entrée dans ce français: armailli n.m. 'vacher' est un " emprunt du français régional au dialecte », tandis que arborisé adj. 'planté d'arbres' est un « type connu dans les dialectes [...] mais comme emprunt au mot du français régional ». Dans de tels cas, la première attestation doit être dédoublée, pour le régionalisme et pour le dialectalisme. De façon systématique est retracée «l'extension géographique du mot dans le reste de la francophonie » (DSR: 30), car si le type lexical est propre à la Suisse romande, comme greffe n.m. 'secrétariat de mairie', ou s'il est présent dans diverses communautés francophones, comme gribiche n.f. 'femme acariâtre' connu également dans certaine régions de France et au Québec, ou comme balan, déverbal de balancer au sens de 'équilibre instable', répandu dans divers français régionaux d'Europe et d'Amérique, ils ne relèvent certainement pas de la même histoire. La perspective historique peut déboucher sur une recherche étymologique neuve, dans le cas des internationalismes qui s'implantent de façon différenciée dans la langue contemporaine, comme autogoal ou bancomat. Le plurilinguisme de la Suisse invite constamment à examiner s'il s'agit ou non d'emprunts sémantiques ou de calques du francoprovençal (donner et frpr. balyi), de l'allemand (à disposition et all. zur Verfügung; dialecte et suisse alémanique Dialekt) ou de l'italien (cornettes, italien cornetti et suisse alémanique Hörnli).

De la même manière, la perspective géolinguistique et historique est constitutive du Dictionnaire des Régionalismes de France (DRF; cf. aussi Glessgen/Thibault 2005), puisqu'il a pour sous-titre: Géographie et histoire d'un patrimoine linguistique. Le commentaire qui, dans chaque article, suit la description proprement lexicographique organise ses informations successivement selon la géographie, l'histoire et l'étymologie. Mais l'étymologie-origine est très loin d'y être prépondérante. Bien sûr le DRF apporte son lot de nouvelles étymologies par rapport au FEW et à l'état de l'art de la lexicologie historique du français, par exemple pour aligot, cotriade, couze, dialecte, fayard ou gadi. Mais son principal objectif en cette matière se limite à établir rigoureusement l'étymon proche, lorsqu'il s'agit d'un emprunt à l'une des langues régionales de France, telles que l'alsacien (baeckeoffe, bibeleskaes, bredele, bretzel), le breton (biniou), le francoprovençal (bachat, barouler, bartavelle, bucler, burler), l'occitan (bacholle, bader, bastide, bastidon, bastonner, bazarette), le gascon (barricot) etc. Le plus souvent, la confrontation de l'aire du régionalisme et de celle de cet étymon proche et leurs datations respectives suffisent à le montrer. Cependant il est quelquefois nécessaire d'argumenter pour décider entre différentes possiblités. La discussion permet de rattacher boge n.f. 'grand sac' au francoprovençal bogi, plutôt qu'à l'ancien et moyen français bouge 'sac de cuir' et à l'ancien occitan boljas n.f.pl. 'grand sac'; bousset n.m. 'barillet' est définitivement considéré comme un emprunt de l'occitan, et non pas du francoprovençal comme on l'a soutenu; buron n.m. 'construction maçonnée servant d'habitat temporaire pendant la belle saison et où se fabrique la fourme' n'est pas un emprunt à l'occitan, comme généralement indiqué, mais une "spécialisation sémantique régionale de mfr. buron 'cabane' ». Lorsque le lexème est une formation d'une variété de français, l'étymologie se limite à dire que bourdelot n.m. 'pomme ou poire enrobée de pâte et cuite au four' vient " du patois normand ", mais l'étymon se résume à une référence au FEW à la dernière ligne de la bibliographie; bredin, -ine adj. et n. 'simple d'esprit' est dit relever "d'un type dialectal » qui est " un dérivé régressif de brediner 'bredouiller' », mais, là aussi, l'étymologie n'est rien d'autre qu'une référence au FEW. Si l'appartenance de deux variantes à un même type (berme / berne) peut être clarifiée, les deux étymons du FEW se réduiront en conséquence à un seul. Mais la recherche étymologique est écartée sans remords lorsqu'elle ne peut aboutir à des conclusions assurées: en tels cas, le mot est déclaré «d'origine inconnue » (baïne), «d'origine obscure » (bigot), ou bien il est référencé dans la partie consacrée aux « inconnus » du FEW (blaude, bouiner), ou bien les doubles classements auxquels il a donné lieu dans le FEW sont signalés sans être tranchés (berte, bistouille), ou 
bien l'étymologisation ne fait l'objet que de renvois à des travaux spécialisés (bouille, boujon, bove, brousiner/broussiner, brousse). On conçoit bien que la question étymologique formelle ne se pose pas pour breton n.m. 'espèce de cépage', mais que c'est la motivation de ce sens qui doit être discutée. La question étymologique est celle de la formation du mot dans le cas de brisaque adj. 'brise-fer': sa notable rétraction contemporaine sur l'aire d'origine par rapport à ses extensions transitoires dans les parlers populaires citadins du $19^{\mathrm{e}}$ siècle permet d'exclure comme point de départ une forme secondaire et excentrée brise-sac et de fournir une explication meilleure que celle du FEW. L'étymologie des régionalismes, c'est d'abord de mettre en lumière la position du mot à l'intérieur du galloroman et la circulation entre les différents parlers et langues, la continuité avec le voisinage belge (ban, berme, bistouille, boujon, brisaque, buquer) ou suisse (ban, barjaquer, berme, bise, blaude, bon, bouille, brési) et les expansions en Amérique du nord (barrer v.tr. 'fermer (une porte) à clef', beurrée, bord, boucaille, bouillée, bugne). Ce programme vient compléter le dispositif mis en place par le FEW pour lequel la question des régionalismes n'était pas centrale.

Dans les dictionnaires de régionalismes de Belgique, la composante étymologique et historique n'a pas été développée comme dans ceux des autres pays francophones. C'est essentiellement l'étymologie-origine qui est mise en œuvre dans ceux qui comportent une notice diachronique (Massion 1987; Francard et al. 2010), la priorité ayant été donnée à la sélection d'une nomenclature jouissant d'une bonne vitalité. De manière significative, les notices diachroniques s'abstiennent de renvoyer au FEW. Mais la perspective historique est seulement différée, car sont annoncées des publications ultérieures qui fourniront «des développements documentés sur l'histoire de chaque mot » (Francard et al. 2010: 13).

\subsection{Lexique spécialisé}

$\mathrm{Du}$ vocabulaire culinaire français qu'étudiait Manfred Höfler et dont il projetait d'écrire un dictionnaire étymologique et historique, la maladie et la mort ne lui auront permis que d'esquisser un lexique des régionalismes culinaires, mis au point par Pierre Rézeau (Höfler / Rézeau 1997) et dont les entrées de large assise ont été reprises pour le DRF, et un lexique du vocabulaire culinaire à valeur adjectivale (Höfler 1996), les adjectifs ou les unités lexicales employées adjectivement dans les dénominations de mets. L'originalité de ce dictionnaire lui vient de ce choix du fonctionnement des unités retenues, qui sont de purs adjectifs (potage printanier/sauce printanière, garniture forestière), ou bien des adjectifs détoponymiques (sauce andalouse, crème anglaise, salade arlésienne), ou bien des traitements adjectivaux de substantifs (pommes boulangère, sauce charcutière, sauce chasseur, pommes paille), de toponymes (potage Compiègne, garniture Louisiane), d'anthroponymes (sauce Béchamel, sauce Talleyrand), de titres d'œuvres artistiques (poire Belle-Hélène, consommé Carmen, salade Francillon, garniture Mascotte), de noms de héros littéraires (pommes Macaire), de noms de batailles (poulet Marengo, rognons Turbigo) ou encore de noms de restaurants (côtelettes Foyot). L'origine déonomastique de bon nombre de ces dénominations lui avait valu d'être, pour une bonne part, ignoré de la lexicographie générale et de faire l'objet d'un véritable légendaire étymologique dans la littérature culinaire. La perspective historique résolument adoptée est seule susceptible d'apporter quelque clarté dans ce domaine. Le principal apport de ce dictionnaire est de fournir une masse d'attestations anciennes documentant la mouvance de ce vocabulaire avant qu'il ne se fixe. Cela permet de manifester la liaison entre un bifteck à la Chateaubriand, un bifteck Chateaubriand, un chateaubriand, un château, des pommes château, la sauce Chateaubriand et la sauce château, en même temps que les échanges entre onomastique et lexique, tandis que la datation de toutes les attestations manifeste le développement de chaque construction. Le repérage des mentions antérieures à la lexicalisation fournit quelquefois un éclairage décisif: qui sait que le Café Riche, fondé en 1804, avait comme spécialités la sole aux crevettes et une sauce 
normande au beurre de homard, n'est plus tenté de voir l'adjectif homonyme dans: la sauce Riche, la sauce à la Riche, la sole à la Riche ou la sole Riche. La connaissance du pâtissier Chiboust, établi rue Saint-Honoré, dans la deuxième moitié du $19^{\mathrm{e}}$ siècle à Paris, éclaire du même coup la dénomination de deux crèmes pâtissières, la crème Chiboust et la crème SaintHonoré. L'attestation au milieu du $19^{\mathrm{e}}$ siècle de volaille Reine Margot / à la Reine Margot décide pour l'étymon entre le nom du personnage historique de la Renaissance et le titre du roman d'Alexandre Dumas, paru en 1845, et de la pièce qui en fut tirée en 1847. De la même manière, le homard Thermidor / à la Thermidor, documenté seulement depuis le début du $20^{\mathrm{e}}$ siècle, doit sa dénomination, plutôt qu'au mois du calendrier révolutionnaire, au titre de la pièce de Victorien Sardou, Thermidor, représentée en 1891. Tous ces historiques établissent la cohérence interne de ces dénominations. Ainsi les écrevisses à la Nantua (1881) expliquent que la sauce Nantua (1883) et la garniture Nantua (1900) comportent du beurre d'écrevisse ou des queues d'écrevisse, mais rien ne justifie que la ville de Nantua soit au départ de cet ensemble. Très souvent le type onomastique est bien identifié, sans que puisse en être déterminé le porteur: la préparation à la Richelieu a été ainsi dénommée " en l'honneur d'un membre de la famille de Richelieu ». L'étymologie est seulement précise quand on peut établir un lien entre la dénomination de la préparation et le nom d'un célèbre cuisinier comme dans le cas de la sauce Dugléré ou du filet de sole Marguery / à la Marguery, ou quand le mets appelé sole Bercy / à la Bercy/sauce Bercy se prépare avec du vin, à une époque où Bercy était le «nom d'un quartier de Paris, qui abrita longtemps le plus vaste marché de vins d'Europe ». Des séries se dessinent comme la garniture Dubarry / à la Dubarry, le potage Fontanges / à la Fontanges, les filets de sole La Vallière / à la La Vallière, les côtelettes Maintenon / à la Maintenon, les soles Waleska / à la Waleska, tous documentés seulement au $19^{\mathrm{e}}$ siècle et même plus souvent à la fin de ce siècle et où l'on reconnaît des noms de favorites royales et impériales célèbres. Les noms de maréchaux d'Empire apparaissent dans des formations presque contemporaines, mais bien postérieures au décès de ces glorieux militaires, dénommant des préparations à la Masséna (1891), à la Murat (1895) et à la Beauharnais (1900) qui comportent toutes des fonds d'artichauts. Des modes spécifiques de dénominations et des enchaînements se laissent deviner grâce à ces historiques. L'introduction d'un secteur lexical nouveau dans la lexicologie historique et étymologique est aussi l'occasion de préciser les relations entre onomastique et lexique et de mettre en lumière le fonctionnement d'un mode de formation lexicale très vivant dans la langue contemporaine.

\section{Conclusion}

Le panorama ici dressé fait ressortir les grandes qualités de la lexicographie historique française: rares sont les domaines qui bénéficient d'une production aussi nombreuse, aussi ambitieuse et aussi aboutie, plus rares encore ceux qui fournissent autant de points de départ méthodologiquement porteurs. Quelques desiderata peuvent cependant être formulés: l'éventail de la lexicographie historique française gagnerait à être complété par des dictionnaires d'emprunts fiables sur le modèle des Orientalia d'Arveiller (1999), un dictionnaire étymologique du lexique scientifique et technique, enfin un dictionnaire historique des unités pragmatiques.

\section{Bibliographie}

\subsection{Dictionnaires, atlas et bases de données}

ALFC $=$ Dondaine, Colette / Dondaine, Lucien: Atlas linguistique et ethnographique de la Franche-Comté (4 volumes). Paris: CNRS Éditions, 1972-1984.

ALW = Remacle, Louis et al.: Atlas linguistique de la Wallonie. Tableau géographique des parlers de la Belgique romane d'après l'enquête de Jean Haust et des enquêtes complémentaires. Liège: Vaillant-Carmanne, 1953-. 
Chauveau 2006- = Chauveau, Jean-Paul (ed.): Französisches Etymologisches Wörterbuch (FEW). Nancy: ATILF, publication électronique < http://www.atilf.fr/few>, 2006-.

Cormier 1999 = Cormier, Yves: Dictionnaire du français acadien. Montréal: Fides, 1999.

DEAF = Baldinger, Kurt et al.: Dictionnaire Étymologique de l'Ancien Français. Québec / Tübingen / Paris: Presses de l'Université Laval / Niemeyer / Klincksieck, 1974-.

DEAFBibl = Möhren, Frankwalt: Dictionnaire étymologique de l'ancien français. Complément bibliographique. Tübingen: Niemeyer, $2007^{3}\left[1974^{1}, 1993^{2}\right]$.

DEAFBiblEl = Möhren, Frankwalt: Dictionnaire Étymologique de l'Ancien Français. Complément bibliographique. Version électronique. Heidelberg: Académie des Sciences de Heidelberg, publication électronique $<$ http://www.deaf-page.de/bibl_neu.htm $>$.

DEAFEl = Städtler, Thomas (ed.): Dictionnaire Étymologique de l'Ancien Français. Version électronique (DEAFplus et DEAFpré). Heidelberg: Académie des Sciences de Heidelberg, $2010-$

DECOI = Bollée, Annegret (ed.): Dictionnaire étymologique des créoles français de l'Océan Indien (4 volumes). Hamburg: Buske, 1993-2007.

DELI = Cortelazzo, Manlio / Zolli, Paolo: Dizionario etimologico della lingua italiana. Bologne: Zanichelli, $1999^{2}\left(1979-1988^{1}\right)$.

DÉRom = Buchi, Eva / Schweickard, Wolfgang (edd.). Dictionnaire Étymologique Roman (DÉRom). Nancy: ATILF, publication électronique $<$ http://www.atilf.fr/DERom>, 2008-.

DMF = Bazin-Tacchella, Sylvie / Martin, Robert / Souvay, Gilles: Dictionnaire du Moyen Français: version DMF 2010. Nancy: ATILF, publication électronique $<$ http://www.atilf.fr/dmf>, 2010.

DOM = Stempel, Wolf-Dieter (ed.): Dictionnaire de l'occitan médiéval. Tübingen: Niemeyer, 1996-.

Dondaine 2002 = Dondaine, Colette: Trésor étymologique des mots de la Franche-Comté. Strasbourg: Société de Linguistique Romane, 2002.

$\mathrm{DRF}=$ Rézeau, Pierre (ed.): Dictionnaire des régionalismes de France. Géographie et histoire d'un patrimoine linguistique. Bruxelles: De Boeck, 2001.

DSR = Thibault, André / Knecht, Pierre: Dictionnaire suisse romand. Particularités lexicales du français contemporain. Genève: Zoé, $2004^{2}\left(1997^{1}\right)$.

FEW = Wartburg, Walther von et al.: Französisches Etymologisches Wörterbuch. Eine darstellung des galloromanischen sprachschatzes (25 volumes). Bonn / Heidelberg / Leipzig-Berlin / Bâle: Klopp / Winter / Teubner / Zbinden, $\quad 1922$ 2002.

Francard et al. $2010=$ Francard, Michel et al.: Dictionnaire des belgicismes. Bruxelles: De Boeck/Duculot, 2010.

Gdf = Godefroy, Frédéric: Dictionnaire de l'ancienne langue française et de tous ses dialectes $\mathrm{du} \mathrm{IX}^{\mathrm{e}}$ au XV $\mathrm{XV}^{\mathrm{e}}$ siècle (10 volumes). Paris: Vieweg, 1881-1902.

GPSR $=$ Gauchat, Louis et al. (ed.): Glossaire des patois de la Suisse romande. Neuchâtel / Paris: Attinger, 1924-.

Höfler 1996 = Höfler, Manfred: Dictionnaire de l'art culinaire français. Étymologie et histoire. Aix-en-Provence: Edisud, 1996.

LEI = Pfister, Max / Schweickard, Wolfgang (edd.): Lessico Etimologico Italiano. Wiesbaden: Reichert, 1979-.

Massion, François: Dictionnaire de belgicismes. Francfort et al.: Peter Lang, 1987.

REW = Meyer-Lübke, Wilhelm: Romanisches Etymologisches Wörterbuch. Heidelberg: Winter, 1930-1935 $35^{3}\left(1911-1920^{1}\right)$.

Poirier $1985=$ Poirier, Claude (ed.): Dictionnaire du français québécois. Volume de présentation. Sainte-Foy: Presses de l’Université Laval, 1985. 
Poirier 1998 = Poirier, Claude (ed.): Dictionnaire historique du français québécois. Monographies lexicographiques de québécismes. Sainte-Foy: Presses de l'Université Laval, 1998.

Robert historique $=$ Rey, Alain (ed.): Dictionnaire historique de la langue française (3 volumes). Paris, Le Robert, $1998^{2}\left(1992^{1}\right)$.

Steinfeld 2007-= Steinfeld, Nadine (ed.): Base des mots fantômes. Nancy: ATILF, publication électronique $<$ http://www.atilf.fr/MotsFantomes $>$, 2007-.

$\mathrm{TL}=$ Tobler, Adolf / Lommatzsch, Erhard: Altfranzösisches Wörterbuch (11 volumes). Berlin / Wiesbaden / Stuttgart: Weidmann / Steiner, 1925-2002.

TLF = Imbs, Paul / Quemada, Bernard (edd.). Trésor de la langue française. Dictionnaire de la langue du $\mathrm{XIX}^{\mathrm{e}}$ et du $\mathrm{XX}^{\mathrm{e}}$ siècle (1789-1960) (16 volumes). Paris: Éditions du CNRS / Gallimard, 1971-1994.

TLF-Étym = Steinfeld, Nadine (ed.): Programme de recherche «TLF-Étym» (révision sélective des notices étymologiques du Trésor de la langue française informatisé). Nancy: ATILF, publication électronique $<$ http://www.atilf.fr/tlf-etym>, 2005-.

TLFi = Imbs, Paul / Quemada, Bernard (edd.). Trésor de la langue française. Dictionnaire de la langue du XIX ${ }^{\mathrm{e}}$ et du $\mathrm{XX}^{\mathrm{e}}$ siècle (1789-1960). Nancy: ATILF, publication électronique $<$ http://atilf.atilf.fr/tlf.htm>, 2004.

\subsection{Monographies et articles}

Andronache $2009=$ Andronache, Marta: Le problème de la continuité en lexicologie historique. In: Studi Italiani di Linguistica Teorica e Applicata 38. 2009, 263-279.

Arveiller 1999 = Arveiller, Raymond: Addenda au FEW XIX (Orientalia). Ed. Max Pfister. Tübingen: Niemeyer, 1999.

Baldinger $1974=$ Baldinger, Kurt: Le FEW de Walther von Wartburg. Introduction. In: Baldinger, Kurt (ed.): Introduction aux dictionnaires les plus importants pour l'histoire du français. Paris: Klincksieck, 1974, 11-47.

Baldinger 1988-2003 = Baldinger, Kurt: Etymologien. Untersuchungen zu FEW 21-23 (3 volumes). Tübingen: Niemeyer, 1988-2003.

Buchi 2005 = Buchi, Éva: Le projet TLF-Étym (projet de révision sélective des notices étymologiques du Trésor de la langue française informatisé). In: Estudis romànics 27. 2005, 569-571.

Buchi 2005-2007 = Buchi, Éva (ed.): Actes du Séminaire de méthodologie en étymologie et histoire du lexique (Nancy/ATILF, année universitaire 2005/2006). Nancy: ATILF, publication électronique $<$ http://www.atilf.fr/methodologie.htm>, 2005-2007.

Buchi 2006 / 2007 = Buchi, Éva (ed.): Actes de la Journée d'étude «Lexicographie historique française: autour de la mise à jour des notices étymologiques du Trésor de la langue française informatisé» (Nancy / ATILF, 4 novembre 2005). Nancy: ATILF, publication électronique <http://www.atilf.fr/atilf/evenement/JourneeEtude/LHF2005>, 2006 / 2007.

Buchi $2007=$ Buchi, Éva: Sur la trace de la pragmaticalisation de l'adverbe toujours ("Voyons toujours l'apport de la linguistique historique"). In: Langue française 154. 2007, 110-125.

Buchi et al. 2010 = Buchi, Éva / Chauveau, Jean-Paul / Gouvert, Xavier / Greub, Yan: Quand la linguistique française ne saurait que se faire romane: du neuf dans le traitement étymologique du lexique héréditaire. In: Neveu, Franck et al. (edd.): Congrès Mondial de Linguistique Française - CMLF 2010. Paris: Institut de Linguistique Française, publication électronique $<$ http://dx.doi.org/10.1051/cmlf/2010025>, 2010, 111-123.

Buchi / Renders à paraître = Buchi, Éva / Renders, Pascale: 46. Gallo-romance I: Historical and etymological lexicography. In: Gouws, Rufus H. et al. (edd.): Dictionaries. An International Encyclopedia of Lexicography. Supplementary volume: Recent developments 
with special focus on computational lexicography. Berlin / New York: de Gruyter, à paraître.

Chambon $1989=$ Chambon, Jean-Pierre: Aspects philologiques et linguistiques dans la refonte du FEW: utilité d'une approche métaphilologique des représentations linguistiques. In: Kremer, Dieter (ed.): Actes du XVIII ${ }^{\mathrm{e}}$ Congrès international de linguistique et de philologie romanes. Tübingen: Niemeyer, 1989, VIII, 218-230.

Chambon $2003=$ Chambon, Jean-Pierre: À propos du Trésor étymologique comtois de Colette Dondaine. In: Revue de Linguistique Romane 67. 2003, 499-532.

Chambon / Billy $1990=$ Chambon, Jean-Pierre / Billy, Pierre-Henri: Pour la mise à jour du $<$ Thesaurus galloromanicus>. Un article d'essai: ATTEGIA. In: Revue de linguistique romane 54. 1990, 61-75.

Chauveau 2005 = Chauveau, Jean-Paul: Remarques sur la dérivation dans les notices historiques et étymologiques du Trésor de la langue française. In: Buchi 2005-2007 $<\mathrm{http} / / / \mathrm{www}$.atilf.fr/atilf/seminaires/Seminaire_Chauveau_2005-11.pdf $>, 22$ pages.

Chauveau / Greub / Seild 2010 = Chauveau, Jean-Paul / Greub, Yan / Seidl, Christian: Französisches Etymologisches Wörterbuch. Eine darstellung des galloromanischen sprachschatzes. Complément. Strasbourg: Société de Linguistique Romane, 2010.

Glessgen, Martin-Dietrich / Thibault, André (edd.): La lexicographie différentielle du français et le Dictionnaire des régionalismes de France. Actes du colloque en l'honneur de Pierre Rézeau pour son soixante-cinquième anniversaire (Strasbourg, Université Marc Bloch, 2022 juin 2003). Strasbourg: Université Marc Bloch, 2005.

Guiraud 1982 = Guiraud, Pierre: Histoire et structure du lexique français. 1: Dictionnaire des étymologies obscures. Paris: Payot, 1982.

Höfler / Rézeau 1997 = Höfler, Manfred / Rézeau, Pierre: Variétés géographiques du français. Matériaux pour le vocabulaire de l'art culinaire. Paris: Éditions du CNRS / Klincksieck, 1997.

Lepelley 1974 = Lepelley, René: Le parler normand du Val de Saire (Manche). Phonétique. Morphologie. Syntaxe. Vocabulaire de la vie rurale. Caen: Musée de Normandie, 1974.

Lerond 1963 = Lerond, Alain: L'habitation en Wallonie malmédienne (Ardenne belge). Étude dialectologique. Paris: Les Belles Lettres, 1963.

Leroy 2009 = Leroy, Sarah: Deonomastica francese nell'ambito del programma TLF-Étym. In: Rivista italiana di onomastica 14. 2009, 614-615.

Möhren $2003=$ [Möhren, Frankwalt]: Dictionnaire étymologique de l'ancien français (DEAF). In: Städtler 2003, 183-194.

Möhren 2000 = Möhren, Frankwalt: "Guai victis ! » Le problème du gu initial roman. In: Medioevo romanzo 24. 2000, 5-81.

Renders 2011 = Renders, Pascale: Modélisation d'un discours étymologique. Prolégomènes à l'informatisation du Französisches Etymologisches Wörterbuch (482 pages et un cédérom). Thèse Université de Liège et Université Nancy 2 (Nancy-Université), 2011.

Rézeau 1976 = Rézeau, Pierre: Un patois de Vendée. Le parler rural de Vouvant. Paris: Klincksieck, 1976.

Städtler 2003 = Städtler, Thomas (ed.): Wissenschaftliche Lexikographie im deutschsprachigen Raum. Heidelberg: Winter, 2003.

Steinfeld 2009 = Steinfeld, Nadine: Bouffer 'manger avec avidité' a-t-il vraiment existé au $\mathrm{XVI}^{\mathrm{e}}$ siècle? Interprétation d'un passage de Clément Marot (Seconde Epistre du Coq en l'Asne, v. 72-79). In: Zeitschrift für französische Sprache und Literatur 119. 2009, 113-127.

Steinfeld / Andronache 2011 = Steinfeld, Nadine / Andronache, Marta: Quoi de neuf du côté de la lexicographie étymologique? La méthode utilisée dans le cadre du projet TLF-Étym pour distinguer les emprunts au latin de l'Antiquité de ceux faits au latin médiéval. In: Estudis romànics 33. 2011, 151-169. 
Thomassen $1997=$ Thomassen, Helga: Zur Behandlung der Datierungen im Dictionnaire historique de la langue française. In: Zeitschrift für romanische Philologie 113. 1997, 3950 .

Wartburg 1953 = Wartburg, Walther von: Organisation et état actuel des travaux relatifs au Französisches Etymologisches Wörterbuch. In: Essais de philologie moderne. Communications présentées au Congrès International de Philologie Moderne, réuni à Liège du 10 au 13 septembre 1951, à l'occasion du LX ${ }^{\mathrm{e}}$ anniversaire des Sections de Philologie germanique et de Philologie romane de la Faculté de Philosophie et Lettres de l'Université de Liège. Paris: Les Belles Lettres, 1953, 97-114.

Wirth $2007=$ Wirth, Aude: L'Anthroponymie de la Lorraine romane du XI ${ }^{\mathrm{e}}$ au XVII siècle. Thèse Université Nancy 2 (Nancy-Université), 2007. 